\title{
Nonlinear Radiative Double-Mode Cepheid Models
}

\author{
A. Fokin \\ Geneva Observatory, CH-1290 Sauverny, Switzerland, and \\ Institute for Astronomy of the Academy of Sciences, Moscow, Russia \\ F. Kienzle, G. Burki \\ Geneva Observatory, CH-1290 Sauverny, Switzerland
}

\begin{abstract}
Robust double-mode pulsation has been found in several nonlinear radiative Cepheid models. This phenomenon has not been previously detected in purely radiative models.
\end{abstract}

\section{Introduction}

The stable double-mode phenomenon has never been observed in radiative nonlinear models (e.g. Kovács 1990; Kolláth et al. 1998). In the course of an extensive hydrodynamical survey of radiative Cepheids, we found several double-mode models, with periods between $3 \mathrm{~d}$ and $11 \mathrm{~d}$, showing robust limit cycles. Their discrete Fourier spectra exhibit only $\mathrm{F}$ and $1 \mathrm{H}$ frequencies and their linear combinations. By "robust" we mean that no significant trend in the amplitudes can be seen during as long as $130,000 \mathrm{~F}$ periods, although some small residual perturbations are observed. The period ratio $P_{1} / P_{0}$ of the double-mode models lies in the interval $0.70-0.72$, and the photometric amplitude ratio $A_{1} / A_{0}$ varies from 0.05 to 0.53 . This result, quite unusual for radiative models, shows that the beat-phenomenon can probably be explained within purely radiative pulsation theory.

\section{Numerical Results}

Two examples are presented here. Figure 1a shows the case of a 3.1-d beat model $\left(L / L_{\odot}=946, M / M_{\odot}=3.875, T_{\text {eff }}=5967 \mathrm{~K}\right)$ after 8600 cycles. No perceptible variation in the spectrum was found during the last 4100 cycles. Note a surprisingly weak, though robust, $1 \mathrm{H}$ Fourier component. Figure $1 \mathrm{~b}$ shows an 8.3-d beat model after 40,000 cycles. No significant trend in the Fourier spectrum towards singly-periodic pulsation was found during the following 40,000 130,000 cycles ( 3000 years in total). Note that the total period of existence of the robust beat phenomenon in this model is already a perceptible fraction of the evolutionary time inside the instability strip (about 12,000 years for the first crossing). 

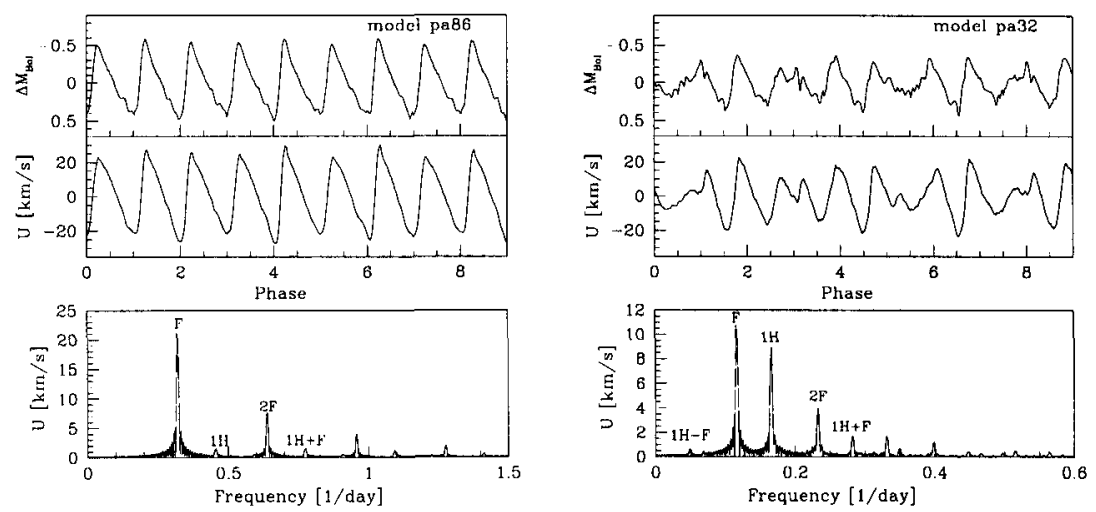

Figure 1. a. Top: light curve for a 3.1-d beat model after 8600 cycles. Middle: velocity curve of the 20th mass zone from the surface after the same time interval. Bottom: the Fourier spectrum of the velocity curve, obtained with a sequence of about 30 periods, with about 40 points per period. The most important peaks are identified. b. Same as Figure 1a but for an 8.3-d beat model after 40,000 cycles.

\section{Discussion and Conclusion}

Are the newly-discovered double-mode limit cycles stable? Although a comparative analysis of the successive Fourier spectra shows their stability over many thousands of cycles, we realise that this is not an absolute stability proof. However, the beat phenomenon found can well be regarded as realistic and observable if the monitoring of the pulsational models would continue long enough compared to the evolutionary time of a Cepheid. In the case of the 8.3- $\mathrm{d}$ model, a run long enough $(130,000 \mathrm{~F}$ cycles!) with respect to the evolutionary time was performed, and the reality of the double-mode pulsation can be postulated.

Why haven't other authors reproduced the beat phenomenon up to now in their radiative models? We can speculate on the basis of differences in the codes and models. Mainly, we use a more advanced treatment of the radiation field (Fokin 1990), which is important for a description of the gas dynamics, not only in the atmosphere (shocks), but also under the photosphere (moving $\mathrm{H}$ ionisation front). Also, the atmospheres of our models have more extended in the density ranges. The outer boundary condition is also different, reducing the shock reflection at the surface.

\section{References}

Fokin, A. B. 1990, Ap\&SS, 164, 95

Kolláth, Z., Beaulieu, J.-Ph., Buchler, J. R., \& Yecko, Ph. 1998, ApJ, 502, L55 Kovács, G. 1990, in NATO ARW Proc., The Numerical Modelling of Nonlinear Stellar Pulsations, Problems and Prospects, ed. J. R. Buchler (Dordrecht: Kluwer), 73 